\title{
Applications of cavity transverse modes in accelerators
}

\author{
V. Volkov \\ Budker Institute of Nuclear Physics (BINP), Lavrentiev Avenue 11, 630090 Novosibirsk, Russia \\ D. Janssen \\ Forschungszentrum Dresden-Rossendorf (FZD), Bautzner Landstrasse 128, 01328 Dresden, Germany
}

(Received 11 February 2008; published 9 June 2008)

\begin{abstract}
Different applications of TE modes in accelerator physics are discussed. In this discussion, the Fourier transform of the squared axial component of magnetic field, $B_{z}{ }^{2}(z)$, plays an important role. If it turns out to be zero, the rf field-particle energy transfer is negligible and the focal length of the TE mode lens is phase independent. Such rf lens focuses continuous beams just as a solenoid. In order to compensate spherical aberrations and emittance growth caused by field nonlinearities of a focusing solenoid, the rf focusing is used also even if the beam space charge is taken into account. If the transform has its maximum, excitations of TE mode resonances and electron beam self-focusing are possible. Furthermore, the rf field of the TE mode can be used to expand the radial acceptance of a FEL for the THz region.
\end{abstract}

DOI: 10.1103/PhysRevSTAB.11.061302

\section{INTRODUCTION}

In most particle accelerators, the electric rf field of a TM mode is applied to accelerate charged particles to higher energies. Besides the TM modes, there is a TE mode, a second class of rf fields, where the magnetic component is applied only along the symmetry axis of the cavity. In superconducting cavities, the maximum axial field, achievable with reasonable surface field strength, is of the order of 0.3-0.4 T. In past years, the application of TE modes has been discussed in connection with superconducting rf guns [1], where the magnetic rf field can replace the static field of a solenoid, used in warm rf guns.

The present paper gives an overview of the possible applications of the TE mode. The paper is arranged as follows: first, the system of coupled equations determining the TE mode and particle motion is derived under assumption of rotational symmetry. Then, different applied aspects are discussed. One of them is the emittance compensation in a superconducting rf gun, which can be reduced completely like that in the case of the static field of a solenoid. It will be also shown that the TE mode can compensate spherical aberration of a static solenoid. The excitation of TE mode resonances and the self-focusing of an electron beam are discussed. We demonstrate also that the TE mode rf field can create a radius dependence of the beam energy, which allows the enhancement of the FEL radiation in the $\mathrm{THz}$ region.

\section{FIELD EQUATION, PARTICLE MOTION, AND ENERGY TRANSFER}

The rf field inside a cavity forms a standing wave. The fields $\bar{E}$ and $\bar{B}$ are solutions of the Maxwell equations with the parallel component of the electric field and the perpendicular component of the magnetic field vanishing at the conducting surface. In what follows, it is useful to repre-
PACS numbers: 41.85. $-\mathrm{p}, 02.60 . \mathrm{Cb}, 85.25 .-\mathrm{j}, 29.20 . \mathrm{Ej}$

sent $\bar{E}$ and $\bar{B}$ as derivatives of vector potential $\bar{A}$ defined by the wave equations [2]:

$$
\Delta \bar{A}-\frac{1}{c^{2}} \frac{\partial^{2} \bar{A}}{\partial t^{2}}=0, \quad \bar{E}=-\frac{\partial \bar{A}}{\partial t}, \quad \bar{B}=\operatorname{rot} \bar{A},
$$

where SI units are used. The TE modes (or magnetic modes) are special solutions of Eq. (1) where (in the axially symmetric case) only the vector components $B_{z}, B_{r}$, and $E_{\varphi}$ differ from zero. In the cylindrical coordinate system, the Maxwell equations for the magnetic modes and the field components of $\bar{E}$ and $\bar{B}$ read as

$$
\begin{gathered}
\frac{\partial B_{r}}{\partial z}-\frac{\partial B_{z}}{\partial r}=\frac{1}{c^{2}} \frac{\partial E_{\varphi}}{\partial t}, \quad E_{\varphi}=-\frac{\partial A_{\varphi}}{\partial t}, \quad B_{r}=-\frac{\partial A_{\varphi}}{\partial z}, \\
B_{z}=\frac{1}{r} \frac{\partial\left(r A_{\varphi}\right)}{\partial r} \quad \text { or } \quad A_{\varphi}(\bar{r}, t)=\frac{1}{r} \int_{0}^{r} B_{z}(\bar{r}, t) r d r \approx \frac{r}{2} B(z, t) .
\end{gathered}
$$

The paraxial approximation $\left.B_{z}(\bar{r}, t) \approx B(\bar{r}, t)\right|_{r=0} \equiv$ $B(z, t)$ and constant trajectory radius of particles in the cavity are assumed hereby. These will also be assumed everywhere below where the sign $\approx$ appears.

Space and time dependencies enter the electric and magnetic components separately, that is why one can write down

$$
\bar{A}(\bar{r}, t)=\bar{A}(\bar{r}) e^{j(\omega t+\alpha)}, \quad \bar{B}(\bar{r}, t)=\bar{B}(\bar{r}) e^{j(\omega t+\alpha)} .
$$

From this it follows that $\bar{A}(\bar{r})=\bar{E}(\bar{r}) / j \omega$, i.e., the vector potential coincides in space with the TE electric field and also has only azimuthal components: $\bar{A}(\bar{r})=A_{\varphi}(\bar{r})$.

The magnetic field components can be expressed from (2) and (3) as

$$
r B_{r}(\bar{r})=-\frac{\partial}{\partial z} r A_{\varphi}(\bar{r}), \quad r B_{z}(\bar{r})=\frac{\partial}{\partial r} r A_{\varphi}(\bar{r}) .
$$

Therefore, the vector 


$$
\left(\begin{array}{l}
B_{r} \\
B_{z}
\end{array}\right)
$$

is tangential to the pattern (equipotential) lines $r A_{\varphi}(\bar{r})=$ const. The solutions of Eq. (1) are labeled through the number of knots of the axial field in $z, r$, and $\varphi$ directions. We will restrict ourselves to the rotationally symmetric $\mathrm{TE}_{0 \mathrm{NM}}$ mode. The pattern lines of $\mathrm{TE}_{011}$ mode in a TESLA cell [3] are shown in Fig. 1. There shown also is the magnetic field distribution on the axis and at the surface of the cavity. The frequency obtained is nearly twice the frequency of the corresponding accelerating mode $\mathrm{TM}_{010}$. In all rf calculations throughout the paper, the code SUPERLANS [4,5] has been used.

In the next step, we will discuss the particle motion in the rf field of the magnetic mode. The equation for an electron motion in an external electromagnetic field $\frac{d}{d t} m \gamma \dot{\bar{r}}=e \bar{E}+e \dot{\bar{r}} \times \bar{B}$ changing for cylindrical coordinate's $z, r$, and $\varphi$ reads for the TE mode field as follows:

$$
\begin{aligned}
m \frac{d}{d t} \gamma \dot{z} & =-e r \dot{\varphi} B_{r}, \\
\gamma & =1 / \sqrt{1-\left(\dot{z}^{2}+\dot{r}^{2}+r^{2} \dot{\varphi}^{2}\right) / c^{2}}, \\
m \frac{d}{d t} \gamma \dot{r} & =m \gamma r \dot{\varphi}^{2}+e r \dot{\varphi} B_{z}, \\
m \frac{d}{d t} \gamma r \dot{\varphi} & =-m \gamma \dot{r} \dot{\varphi}+e E_{\varphi}+e\left(\dot{z} B_{r}-\dot{r} B_{z}\right) .
\end{aligned}
$$
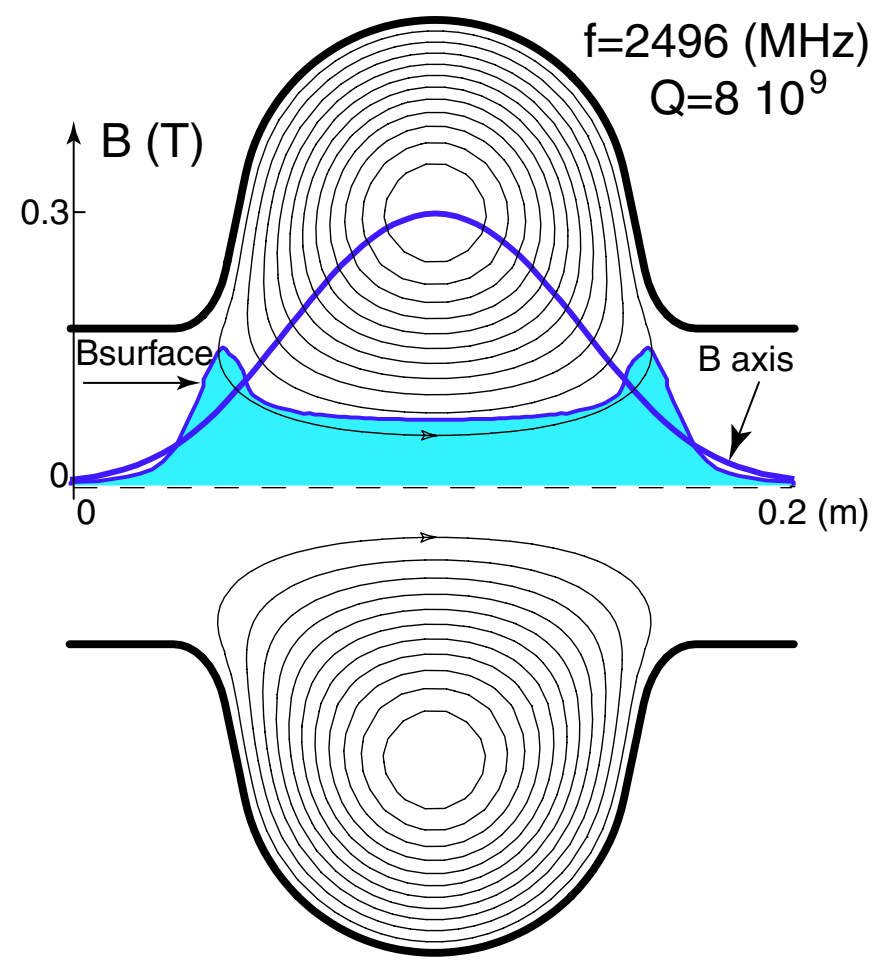

FIG. 1. (Color) Field pattern lines, axis, and surface field of the TESLA cell.
By using Eq. (2) and taking into account that $\frac{d}{d t} \equiv \frac{\partial}{\partial t}+$ $\dot{z} \frac{\partial}{\partial z}+\dot{r} \frac{\partial}{\partial r}$, we can rewrite final equation (5) as follows:

$$
\frac{d}{d t}\left(m \gamma r^{2} \dot{\varphi}\right)=e r E_{\varphi}+e r\left(\dot{z} B_{r}-\dot{r} B_{z}\right) \equiv \frac{d}{d t}\left(-e r A_{\varphi}\right) .
$$

It is possible to integrate the equation for the angular component of the particle momentum $P_{\varphi}=m \gamma r \dot{\varphi}$, and then we obtain the first identity:

$$
\begin{aligned}
P_{\varphi}(\bar{r}, t) & =-e A_{\varphi}(\bar{r}, t)+\mathrm{const} / r \\
& \approx-e \frac{r}{2} B(z, t)+\mathrm{const} / r,
\end{aligned}
$$

const $=e A_{\varphi 0} r_{0}+P_{\varphi 0} r_{0}$, where $P_{\varphi 0}, r_{0}$, and $A_{\varphi 0}$ are the parameters at the cavity entrance. We put below const $=0$.

In Eq. (7), we have obtained the precise and approximate equations. The Busch theorem [6] is a special case of approximated Eq. (7) but here it is extended to the case of a nonstatic or time-dependent electromagnetic field $(\omega \neq 0, \alpha \neq 0)$ :

$$
\dot{\varphi}(\bar{r}, t)=-\frac{e}{m} \frac{1}{\gamma r} A_{\varphi}(\bar{r}, t) \approx-\frac{e}{m} \frac{1}{2 \gamma} B(z) \cdot e^{j(\omega t+\alpha)} .
$$

In the next step, if we replace in Eq. (5) $\dot{\varphi}$ with Eq. (8) (const $=0$ is assumed), we obtain

$$
\begin{aligned}
& \frac{m^{2}}{e^{2}} \frac{d}{d t} \gamma \dot{z}=-\frac{1}{2 \gamma} \frac{\partial A_{\varphi}(\bar{r}, t)^{2}}{\partial z}, \\
& \frac{m^{2}}{e^{2}} \frac{d}{d t} \gamma \dot{r}=-\frac{1}{2 \gamma} \frac{\partial A_{\varphi}(\bar{r}, t)^{2}}{\partial r} .
\end{aligned}
$$

These equations are identical to the equation for an electron, moving in a static magnetic field, where the square of static vector potential $A_{\varphi}^{2}(\bar{r})$ is replaced by time-dependent $A_{\varphi}^{2}(\bar{r}, t)$. For rf field (3), we have

$$
\begin{aligned}
A_{\varphi}^{2}(\bar{r}, t) & =A_{\varphi}^{2}(\bar{r}) \cos (\omega t+\alpha)^{2} \\
& =A_{\text {stat }}^{2}(\bar{r})+\frac{1}{2} A_{\varphi}(\bar{r})^{2} \cos (2 \omega t+2 \alpha), \\
A_{\text {stat }} & =\frac{A_{\varphi}}{\sqrt{2}} .
\end{aligned}
$$

The particle trajectories in this field depend on the phase $\alpha$ of the rf mode. Therefore, the magnetic field focuses each bunch of the beam, entering the cavity at different phases, with different strength. At the same time, the static part of the rf acts in the same way as a static field with the lower strength of the effective rf amplitude. Such an rf lens is able to focus continuous electron beams. This issue will be discussed thoroughly in the next section.

Let $v$ be the nonazimuthal component of particle velocity, such that $v^{2}=\dot{z}^{2}+\dot{r}^{2}$. The insertion of $\dot{\varphi}(\bar{r}, t)$ from (8) to $\gamma$ in Eq. (5) gives $\gamma=$ $1 / \sqrt{1-v^{2} / c^{2}-\left[A_{\varphi}(\bar{r}, t) e / m c\right]^{2} / \gamma^{2}}$ and then 


$$
\gamma(\bar{r}, t)=\sqrt{1+\left[A_{\varphi}(\bar{r}, t) e / m c\right]^{2}} / \sqrt{1-v^{2}(\bar{r}, t) / c^{2}} .
$$

Now we analyze the equations (9). Multiplying both sides of upper Eq. (9) by $\gamma \dot{z}$, lower one by $\gamma \dot{r}$, and summing them, we obtain

$$
\begin{aligned}
\frac{1}{e^{2}} \frac{d}{d t}(m \gamma v)^{2}= & -\dot{z} \frac{\partial A_{\varphi}(\bar{r}, t)^{2}}{\partial z}-\dot{r} \frac{\partial A_{\varphi}(\bar{r}, t)^{2}}{\partial r}-\frac{\partial A_{\varphi}(\bar{r}, t)^{2}}{\partial t} \\
& +\frac{\partial A_{\varphi}(\bar{r}, t)^{2}}{\partial t} .
\end{aligned}
$$

Integrating Eq. (12) over $t$ in the same manner applied for Eq. (7), we obtain the second identity for the nonangular component of the particle momentum $P_{v}=m \gamma v$ :

$$
\begin{aligned}
& P_{v}^{2}(\bar{r}, t)-P_{v 0}^{2}=e^{2}\left\lfloor A_{\varphi 0}^{2}-A_{\varphi}(\bar{r}, t)^{2}+\Delta(\bar{r}, t)\right\rfloor, \\
& \Delta(\bar{r}, t)=\int_{0}^{t} \frac{\partial A_{\varphi}^{2}(\bar{r}, \tau)}{\partial \tau} d \tau=j e^{j 2 \alpha} \omega \int_{0}^{t} A_{\varphi}^{2}(\bar{r}) e^{j 2 \omega \tau} d \tau .
\end{aligned}
$$

For a static solenoid $\Delta \equiv 0, \gamma \equiv \gamma_{0}$, and if $A_{\varphi 0}=0$, a slight rearrangement of terms gives

$$
v(\bar{r}, t)=\sqrt{v_{0}^{2}-\left[A_{\varphi}(\bar{r}, t) e / \gamma m\right]^{2}} .
$$

Now let us have a look at the particle energy equation $W=m c^{2} \gamma$. Using (7) and (13) and the relativistic relation $|\bar{P}|^{2} \equiv P_{v}^{2}+P_{\varphi}^{2}=W^{2} / c^{2}-m^{2} c^{2}$, we obtain the third identity:

$$
W^{2}(\bar{r}, t)=W_{0}^{2}+\Delta(\bar{r}, t) e^{2} c^{2} / 2
$$

or

$$
\begin{aligned}
\Delta W(\bar{r}, t) / e & \approx \Delta(\bar{r}, t) e /\left(2 m c^{2} \gamma\right) \\
& \approx j e^{j 2 \alpha} \frac{e \omega}{8 m \gamma} \int_{0}^{t} r^{2} B^{2}(z) e^{j 2 \omega \tau} d \tau .
\end{aligned}
$$

The condition $\left.\Delta\right|_{t \rightarrow \infty}=0$ means that the final energy is not changed. One can note that, using (2) and (8), Eq. (14) of energy alteration $\Delta$ can be rewritten in the following way: $\Delta(\bar{r}, t)=2 \frac{m}{e} \int_{0}^{t} \gamma r \dot{\varphi} E_{\varphi} d \tau=2 \frac{m}{e} \int_{0}^{t}(\dot{\bar{r}} \cdot \bar{E}) d \tau$, i.e., it possesses that the energy transfer holds only in particle-Efield interactions, which is corresponding to the conclusions of classical physics [2].

We note that the accuracy of numerical calculations of particle dynamics in TE mode fields by any tracking code can be easily verified with the assumption of Eq. (8): the azimuthal component of particle velocities must be zero if the particles initially having zero azimuthal velocity in free space are left out of TE field. In all dynamics calculations throughout the paper, the unique code ASTRA [7] has been used. Its accuracy is good enough. As a rule it becomes better by an order if the cylindrical system of coordinate is applied instead of the Cartesian one (it is predicted by MATHCAD simulations).

\section{COMPENSATION OF EMITTANCE GROWTH IN A SUPERCONDUCTING RF GUN}

For generation of electron bunches with a charge of $1 \mathrm{nC}$ and an emittance of $\sim 1 \mathrm{~mm}$ mrad laser driven rf photocathode guns are applied [8]. These guns are based on normal conducting cavities and operated only in a pulsed rf mode. In order to minimize the emittance, the beam is focused inside the cavity by two solenoids. Application of superconducting cavities allows the continuous mode operation, necessary for high average currents. In these cavities, one has to replace the static magnetic field of the solenoids by the magnetic field of an additional $\mathrm{rf}$ mode. Numerical simulations have shown that the magnetic rf focusing by the TE mode discussed above compensate the emittance growth [9] and the results depend very weakly on the phase $\alpha$ of the TE mode.

In the following, we will discuss the phase dependence of the magnetic rf focusing in more detail. A simple consideration shows that the focal length $F$ of the magnetic lens, which is generated by the axial field $B(z)$, is given by $F=-r_{0} /\left.\frac{d r}{d z}\right|_{z=\infty}$. With an account for Eq. (9) we rewrite this definition as $1 / F=-\left.\frac{1}{r_{0}} \frac{\gamma \dot{r}}{\gamma \dot{z}}\right|_{z=\infty}=-\frac{1}{r_{0} \dot{z}_{0} \gamma_{0}} \int_{0}^{\infty} \frac{1}{\gamma} \times$ $\frac{\partial A_{\varphi}^{2}(\bar{r}, t)}{\partial r} d t$ and for $r \approx$ const, we obtain the approximation $(\dot{z} \equiv \beta c)$ :

$$
\begin{gathered}
1 / F \approx \frac{1 / c}{2 \gamma_{0}^{2} \beta_{0}} \int_{0}^{\infty} B^{2}(z, t) \cos (\omega t+\alpha)^{2} d t \\
=\frac{1 / c^{2}}{2 \beta_{0}^{2} \gamma_{0}^{2}} \int_{0}^{\infty}\left(\frac{B(z)}{\sqrt{2}}\right)^{2} d z+\frac{1 / c}{4 \gamma_{0}^{2} \beta_{0}} \\
\times\left|\int_{0}^{\infty} B^{2}(z) e^{j 2 \omega t} d t\right| e^{j(2 \alpha+\Phi)}, \\
\Phi=\arg \left(\int_{0}^{\infty} B^{2}(z) e^{j 2 \omega t} d t\right) .
\end{gathered}
$$

The first term of (18) corresponds to the focal strength of static lens with $B_{\text {stat }}=B_{z} / \sqrt{2}$. The second one characterizes the phase dependence of the rf lens. This integral is proportional to the Fourier transform of $B^{2}(z)$. The frequency dependence of the Fourier transform decreases with the frequency increase and has a number of maxima alternating by minima. The minima are equal to zero if the distribution $B(z)$ is symmetrical, i.e. $B\left(z_{0}+z\right)=B\left(z_{0}-\right.$ $z)$. This consideration for the resonance frequency $\omega$ of the TE mode leads to the following:

$$
\left|\int_{0}^{\infty} \frac{\partial A_{\varphi}^{2}(\bar{r})}{\partial r} e^{j 2 \omega t} d t\right| \approx r_{0}\left|\int_{0}^{\infty} B^{2}(z) e^{j 2 \omega t} d t\right|=0 .
$$

From Eq. (20) and the approximation discussed before, it follows that the energy transfer $\Delta W$ given by Eq. (17) is equal to zero. In this way, the phase independence of the focal length and the zero energy transfer between the 
magnetic rf field and particle energy coincide with the case of static magnetic field completely. The particle trajectories inside the TE mode field can differ from the corresponding trajectories in the solenoid, but outside they agree with a good approximation.

We would like to remark that there exist two important properties of $\mathrm{rf}$ lenses following from the Fourier transformation.

(i) One can always design a focusing cavity having those resonant frequencies of TE modes, that the phase dependent part of focusing strength, Eq. (20), is equal to zero and rf lens is operated as a static lens, i.e., such rf lens is able to focus continuous beams.

(ii) Those TE modes can always be chosen in such a way that resonant frequencies are large enough to obtain the weak phase dependence.

Now we will design a "phase independent" TE mode cavity which is able to focus continuous beams. In the first step, we define an axis field $B(z)$. Then we calculate the Fourier transform $D(\omega)=\left|\int_{-\infty}^{\infty} B^{2}(c t) e^{j 2 \omega t} d t\right|$ and determine the roots $\omega_{0}$ of $D$.

Let us give a simple example of a pillbox cavity. For the axial field we assume

$$
B(z)=\begin{array}{cc}
B_{0} \cos (\pi z / L) & \text { for }|z| \leq L / 2 \\
0 & \text { for }|z| \geq L / 2
\end{array}
$$

The Fourier transform of the field is $D(\omega)=\frac{B_{0}^{2}}{2 \omega} \times$ $\frac{1+(\omega L / \pi c)^{2}}{1-(\omega L / \pi c)^{2}}|\sin (\omega L / c)|$ and the lowest root $\omega_{0}$ is equal to $2 \pi c / L$ or $k=\omega_{0} / c=2 \pi / L$. The axial field of Eq. (21) corresponds to a pillbox cavity with the length $L$ and radius $R$ in the $\mathrm{TE}_{110}$ mode. The frequency of the pillbox cavity is given by $\omega_{p b}=c \sqrt{(\pi / L)^{2}+\left(p_{01}^{\prime} / R\right)^{2}}$, where $p_{01}^{\prime}$ is the first root of the Bessel function $J_{1}$. Now we put $\omega_{p b}$ equal to the root $\omega_{0}$ of the Fourier transform and obtain the relation

$$
\frac{R}{L}=\frac{p_{01}^{\prime}}{\pi \sqrt{3}}, \quad B_{z}=B_{0} \cos \left(\frac{k}{2} z\right) .
$$

When this ratio between the radius and the length of the pillbox cavity holds, the focal length defined in Eq. (18) is phase independent and the energy transfer $\Delta W$ between field and particles is zero. In the case of a realistic field distribution, the reconstruction of the cavity shape from the axis field $B(z)$ is more difficult. In the first step, one has to calculate the axial field and frequency for a given cavity shape. Subsequently, one changes the shape radius for the constant cell length in such a way that a root of the Fourier transformed axial field agrees with the cavity frequency. This procedure has been applied in [9]. In this paper, the beam properties of a superconducting rf gun have been optimized using three different cavity shapes. The magnetic focusing has been applied by the rf field of a TE mode inside and behind the accelerating cavities. Figures 2-4 show the shapes of the cavities, the field pattern, and the

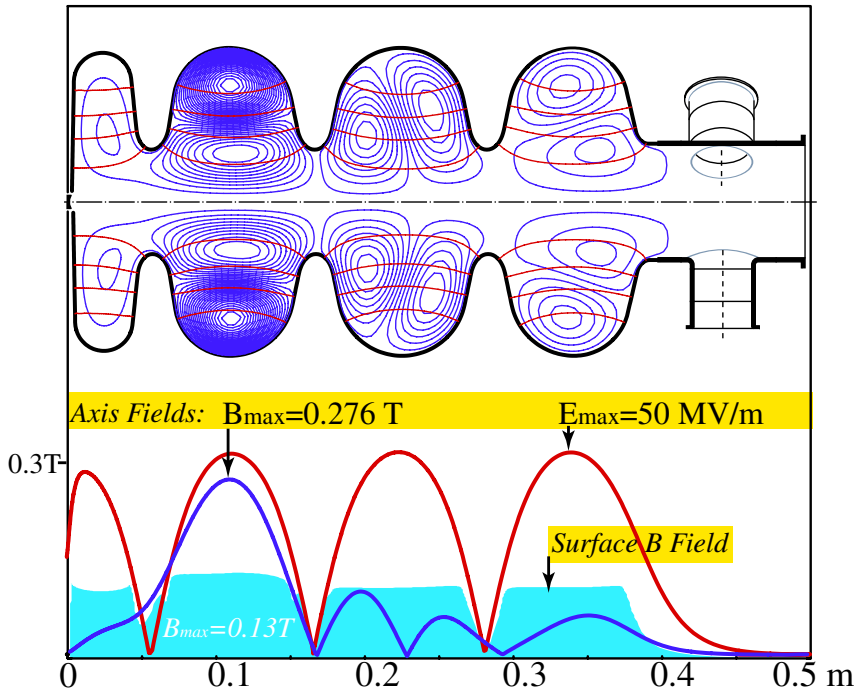

FIG. 2. (Color) Shape, field pattern, the axis, and surface fields of $3 \frac{1}{2}$ cell superconducting cavity of the rf gun having $\mathrm{TE}_{021}$ mode with the frequency of $3781 \mathrm{MHz}$.

axis and surface fields of the corresponding TE modes. In all cases, the optimal axis field has its maximum at $\sim 0.3 \mathrm{~T}$ and the peak of the surface field is below the critical quench value of a superconducting cavity.

The Fourier transform of the TE mode axis fields together with the corresponding frequencies are given in Figs. 5 and 6 . In the case of $3 \frac{1}{2}$ cells, the TE mode and

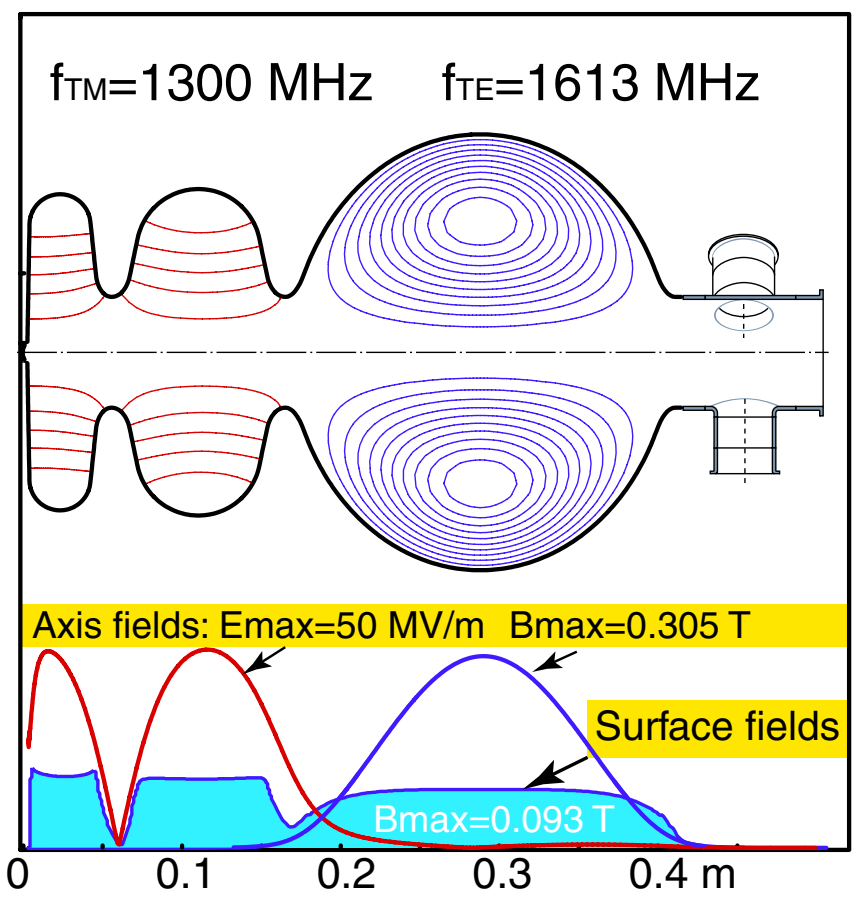

FIG. 3. (Color) Shape, field pattern, the axis, and surface fields of $1 \frac{1}{2}$ cell superconducting cavity of the rf gun with the separated focusing $\mathrm{TE}_{011}$ mode having the frequency of $1613 \mathrm{MHz}$. 


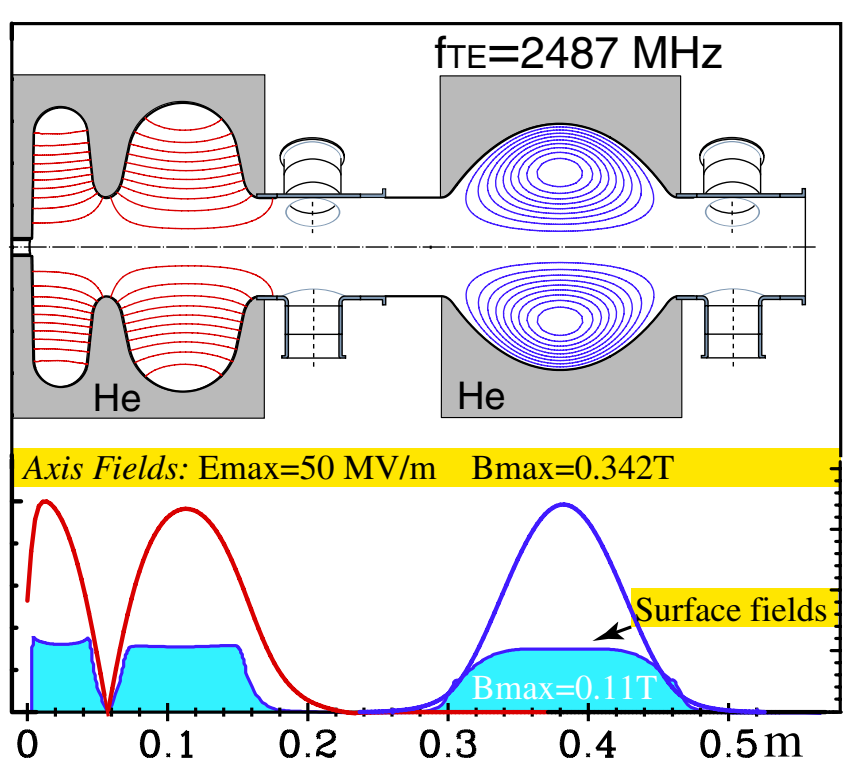

FIG. 4. (Color) Shape, field pattern, the axis, and surface fields of $1 \frac{1}{2}$ cell superconducting cavity of the rf gun with the separated focusing cavity having $\mathrm{TE}_{011}$ mode frequency of $2487 \mathrm{MHz}$.

the acceleration TM mode are in the same cavity. Therefore, the cavity shape is a compromise between the optimal electric TM axis field near the cathode and the phase independence of the TE mode axis field. The Fourier transform minima of this TE mode axis field are small, but not zero. This occurs due to the nonsymmetrical distribution of $B^{2} / \gamma$ in respect with its center $z_{0}: B^{2}\left(z_{0}+z\right) / \gamma \neq$ $B^{2}\left(z_{0}-z\right) / \gamma$. For the bunch charge of $2.5 \mathrm{nC}$ and bunch length of $21 \mathrm{ps}$, the transverse emittance of $0.99 \mathrm{~mm} \mathrm{mrad}$ was obtained [9]. This is an excellent value, which changes only by $\pm 4 \%$, during phase variation of the TE mode field. We would like to note that, in this case, the energy $\gamma$ and velocity $v$ are time dependent due to acceleration of elec-

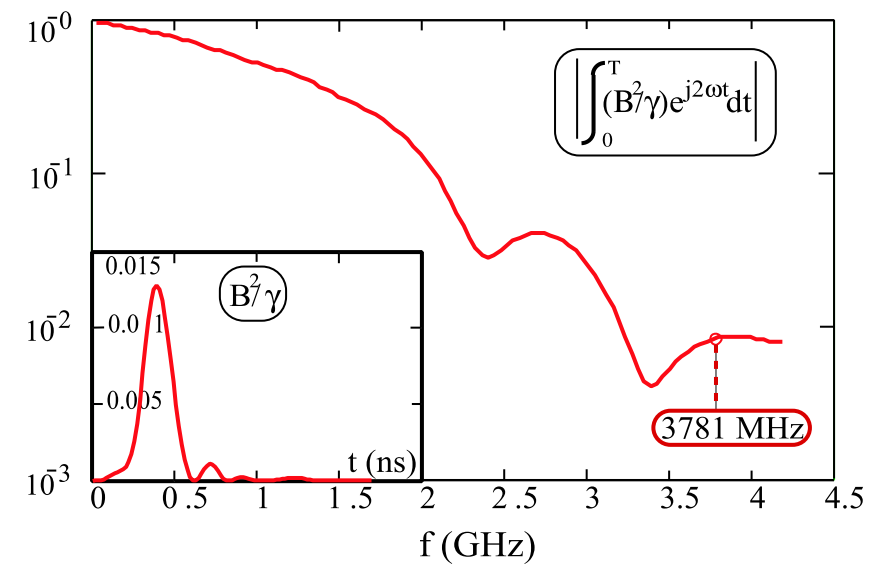

FIG. 5. (Color) $\mathrm{TE}_{021}$ mode axis fields, frequencies, and the Fourier transform for the $3 \frac{1}{2}$ cell cavity [9]. In this case, energy $\gamma(t)$ and velocity $v(t)$ are time dependent.

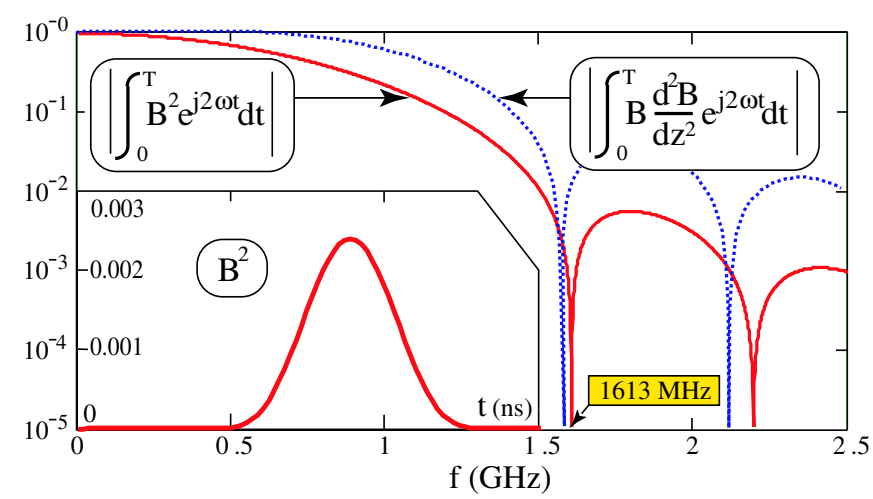

FIG. 6. (Color) TE mode axis fields, frequencies, and the Fourier transform for the $1 \frac{1}{2}$ cell $\mathrm{rf}$ gun [9] with separated focusing $\mathrm{TE}_{011}$ mode having the resonance of $1613 \mathrm{MHz}$ shown in Fig. $3(\gamma=$ const). The dotted line dependence is analyzed in the next chapter.

trons and one has to incorporate these values in the integrands of Eqs. (18)-(20).

For the $1 \frac{1}{2}$ cell cavities, the TM and TE modes are separated and we have determined the shape of the TE mode cell by the procedure discussed before. In this case, the transverse emittances are $1.13 \mathrm{~mm} m r a d$ and $1.46 \mathrm{~mm}$ mrad, respectively, and the phase dependence of these values is $\pm 0.3 \%$. This value is within the numerical noise of the applied tracking calculation. The slightly larger emittance for the $1 \frac{1}{2}$ cell cavities can be explained by a larger distance of the magnetic field maximum to the cathode in these cases.

We close this section by recapitulating the advantages of the TE mode application with respect to the solenoid field close to the superconducting rf gun: (i) The magnetic field maximum can be placed near the cathode, which improves compensation of the emittance growth. (ii) No phase stabilization and frequency tuning is needed; only amplitude stabilization of the TE mode is necessary. (iii) The necessary rf power is on the order of several tens watts, which can be generated by a semiconductor amplifier. (iv) No additional shielding is needed; the hole of solenoid shielding around the beam pipe must be as small as possible to place the solenoid near the superconducting cavity. This leads to an increase in the $B$ field nonlinearities and emittance growth. (v) No additional mechanical adjustment is necessary. (vi) The application of a TE mode focusing is less expensive than the focusing by a solenoid.

\section{FIELD NONLINEARITY COMPENSATION}

It is known [10] that the nonlinearities of the static $B$ field near the axis similarly to the nonlinearities of the acceleration rf field [11,12] is a reason of the spherical aberration and emittance growth. This always has a negative influence on the beam quality and could not be compensated in any combination of static lenses or rf cavities 
separately if the space charge is not taken into account. Because of the spherical and chromatic aberrations, the resolution of electron microscopes is improved only by two orders compared to light microscopes, but it seems to be of five orders according to the ratio of the light wavelength to the electron one. We shall compare the nonlinearities of static solenoid fields and the rf field of TE modes and then analyze the differences to its applying for the compensation of spherical aberration and the emittance growth. In this problem the space charge is taken into account.

Let us define the axial symmetric $B$ fields through the nonlinearity factor $(b)$ as follow:

$$
B_{z}(\bar{r})=B(z)+b(z) \cdot r^{2}, \quad B(z)=\left.B_{z}(\bar{r})\right|_{r=0} .
$$

By using Eq. (2) we obtain

$$
\begin{aligned}
b(z) & =-\frac{k^{2}}{4} B(z)-\frac{1}{4} \frac{\partial^{2} B(z)}{\partial z^{2}}, \\
E_{\varphi}(\bar{r}) / j \omega & =-\frac{r}{2} B(z)-\frac{r^{3}}{4} b(z), \\
B_{r}(\bar{r}) & =-\frac{r}{2} \frac{\partial B(z)}{\partial z}-\frac{r^{3}}{4} \frac{\partial b(z)}{\partial z} .
\end{aligned}
$$

In the first terms of Eq. (24), the axis distribution of nonlinearity factor $b(z)$ is obtained. We assume here that the independent variation of the $k$ and field distribution $B(z)$ are possible in some limits. In a simple static solenoid $(k=\omega / c \equiv 0)$, at a maximum of $B$ field $\left(z \approx z_{0}\right)$, the factor $b\left(z_{0}\right)$ always has a positive value (for $B>0$ ):

$$
b_{\text {solenoid }}\left(z_{0}\right)=-\frac{1}{4} B^{\prime \prime}>0, \quad B^{\prime \prime}=\frac{\partial^{2} B(z)}{\partial z^{2}} .
$$

But for the rf field of TE mode this factor becomes a negative value due to the addend with the $k^{2}$ coefficient. Since the dynamics equations depend on $A^{2} \approx(r / 2)^{2} B^{2}$ field (see Sec. II), we must consider the nonlinearities of the $B^{2}$ field, i.e. $B^{2}(\bar{r}) \approx B^{2}(z)+r^{2} 2 B(z) b(z)$. For the example of the TE field presented in Fig. 6, this factor is $2 B b_{\mathrm{TE}} / B_{\max }^{2}=-43001 / \mathrm{m}^{2}$, but for the static solenoid within the same $B$ field distribution and $k=0$, it is $2 B b_{\text {sol }} / B_{\max }^{2}=10501 / \mathrm{m}^{2}$. The differences between the static and rf nonlinearities will be clearer if we consider their distributions along the axis. The distribution of factors $2 B(z) b(z) / B_{\max }^{2}$ on axis for the TE field of Fig. 6 and for the solenoid field having the same $B(z)$ distribution with $k=0$ are presented in Fig. 7 .

A reasonable question occurred: Could the phase independence in such a field for any $r$ be proved when Eq. (20) is completing, i.e.

$$
\left|\int_{0}^{\infty} B(z) b(z) e^{j 2 \omega t} d t\right|=\left|\int_{0}^{\infty} B B^{\prime \prime} e^{j 2 \omega t} d t\right|=0 ?
$$

For the above-mentioned pillbox cavity having cosine distribution of Eq. (22), the phase independence completes always, $B^{\prime \prime}=\left(k^{2} / 4\right) B$, but for another cavity, the optimal

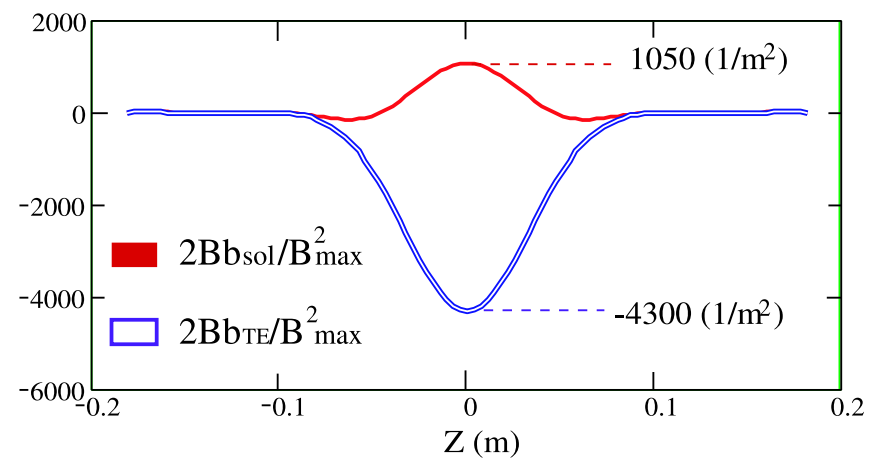

FIG. 7. (Color) Distributions $2 B(z) b(z) / B^{2}{ }_{\max }$ of the TE lens and the static solenoid fields of Fig. 6.

shape must be found in order to complete Eqs. (20) and (26). In Fig. 6, the Fourier transform of the nonlinearity part $r^{2} 2 B(z) \partial^{2} B(z) / \partial z^{2}$ for the field distribution of TE cavity Fig. 3 is depicted. In the resonance frequency $1613 \mathrm{MHz}$ it is small enough $(<0.01)$, i.e., the nonlinearity compensation will be held with an accuracy of $\pm 1 \%$. This is correct for the TE cavity of Fig. 4 also.

It would be logical to predict that the rf lens has those aberration factors having the opposite sign to the static solenoid one, and in the combination of a TE lens and a static solenoid their aberration factors could be compensated to zero. The emittance growth caused by the nonlinearities of these fields must be compensated also.

These predictions are reconfirmed by the numerical ASTRA simulations. In Figs. 8-11, we show the calculated trajectories of $3 \mathrm{MeV}$ electron beams emitted from a dotted source and focused by the static solenoid, by the rf lens of Fig. 4 (both having the field distributions like Fig. 6), and by its combination. The initial emittance just after the source is equal to zero, but due to these nonlinearities, the emittance growth after both lenses up to 4.1 and $25.4 \pi \mathrm{mm}$ mrad (see Figs. 8 and 9) occurred. The focal rms spot sizes that are the resolution defined of scanning microscopes [13] are 0.08 and $0.63 \mathrm{~mm}$, respectively.

The $B_{\max }$ amplitude of TE mode in both lens combinations of Figs. 10 and 11 is optimized to compensate the aberrations completely. In the first combination of Fig. 10, the rf lens is disposed into the static solenoid. The focal strengths and focal rms spot sizes here depend on the rf phase of TE mode because Eq. (20) for the field sum is not adjusted to zero; therefore it can be used for bunched beams only and with phase adjusting systems applied. On the contrary, the second combination in Fig. 11 is insensitive to the phase. The calculated resolutions and emittances in both lens combinations in Figs. 10 and 11 are practically zero within the range of numerical calculation accuracy.

Unfortunately, there is a resolution growth due to chromatic aberrations that depend on the factor $\beta^{2} \gamma^{2}$ mentioned in Eq. (18). In Figs. 10 and 11, we depict these resolutions when the electron source has the rms energy spread of $\sigma_{E} / E=0.1 \%$. For this reason, the resolution of 


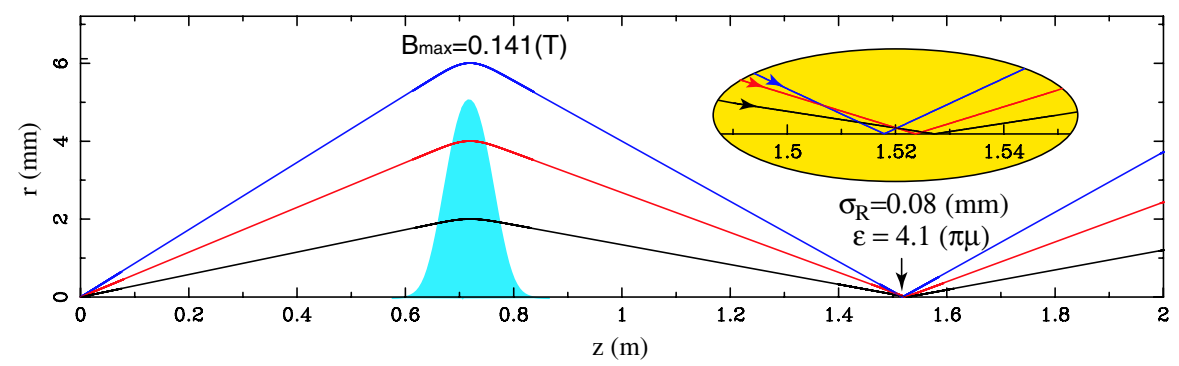

FIG. 8. (Color) Probe particle trajectories of the beam in the static solenoid. $\varepsilon$ is normalized rms transversal emittance after the lens; $\sigma_{R}$ is rms spot size in the focal point.

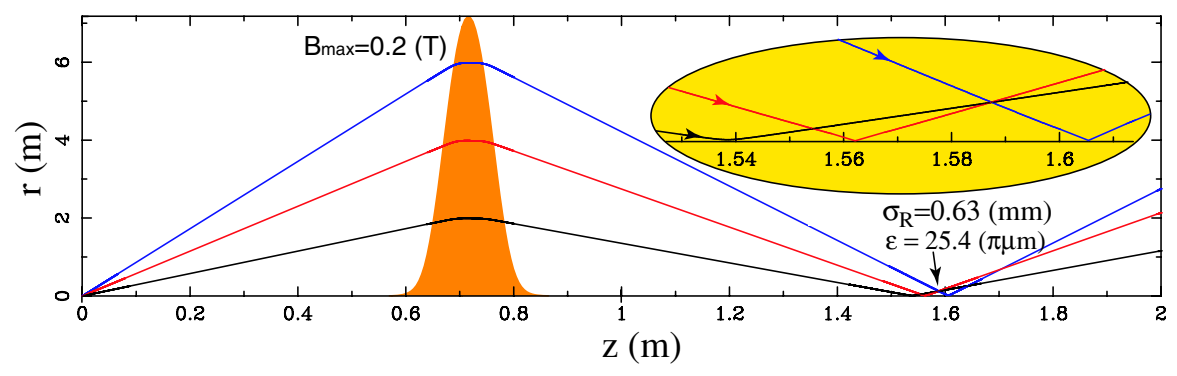

FIG. 9. (Color) Probe particle trajectories of the beam in the rf lens $(\omega / 2 \pi=2.47 \mathrm{GHz})$.

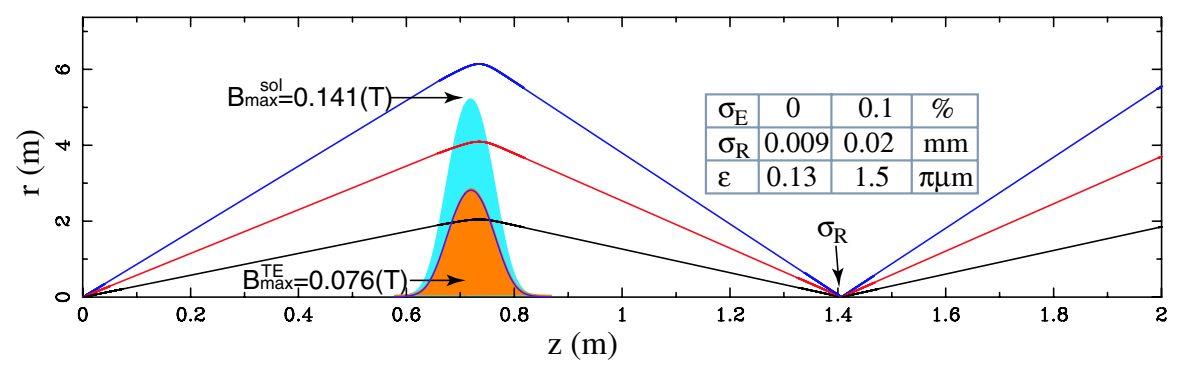

FIG. 10. (Color) Probe particle trajectories of the beam in the combination of the static solenoid and rf lens. $\sigma_{E}$ is rms energy spread just after the source, before the lens. $\omega / 2 \pi=2.47 \mathrm{GHz}$.

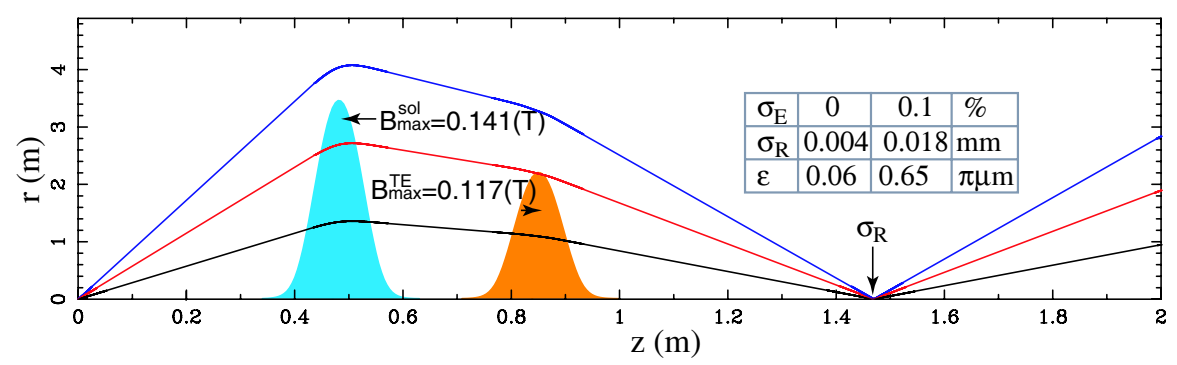

FIG. 11. (Color) Probe particle trajectories of the beam in the combination of the static solenoid and $\mathrm{rf}$ lens $(\omega / 2 \pi=2.47 \mathrm{GHz})$.

recent available electron microscopes cannot be less than a few of nanometers, even if they will have zeroth spherical aberrations [13].

We note here that, by such double combinations of the rf and solenoid lenses, we can focus those beams having larger radius, i.e., more current beams could be focused qualitatively. Such doublets are compatible for transport- ing beams (continuous included) to long distances where the periodically placed focusing is applied many times.

In order to confirm it by simple equations, let us consider those models of such a doublet presented in Fig. 11, in which the spatial charge is taken into account. Ideally, the continuous beam here must have a zeroth emittance in both (entrance and focal point) cross sections and equal trans- 
verse sizes, i.e., all trajectories must be parallel to the axis and the particles must have zero transversal momenta in both cross sections (beam waist) $[14,15]$. This occurs if the integrated transversal momentum of focusing forces on the distance from the entrance up to the focal point is compensated by the space charge forces.

Let us consider for all trajectories the approximation case with $r(z) \approx$ const. If the beam has the uniform transverse distribution of the current density $\left(j_{o}\right)$ then space charge forces acting on the particle with $r$ coordinate is $2 \pi r j_{0} /\left(I_{0} c \beta^{2} \gamma^{2}\right) \quad[10], \quad$ where $I_{o}=4 \pi \varepsilon_{o} m_{o} c^{3} / e=$ $17000 \mathrm{~A}$. After adding this in the last line of Eq. (9), using Eq. (2), and assuming $d / d t=\dot{z} d / d z$, TE and solenoid fields are separated from each other in space, we obtain

$$
\begin{aligned}
\frac{d}{d z}\left(\beta \gamma r^{\prime}\right)= & -\left(\frac{e}{2 m c}\right)^{2} \frac{r}{\beta \gamma} B_{s}^{2}(z, r) \\
& -\left(\frac{e}{2 m c}\right)^{2} \frac{r}{\beta \gamma} B_{\mathrm{TE}}^{2}(z, r, t)+\frac{2 \pi j_{0}}{I_{0} c} \frac{r}{\beta^{2} \gamma^{2}},
\end{aligned}
$$

where $r^{\prime}=d r / d z$ and $B_{s}(z, r), B_{\mathrm{TE}}(z, r)$ is correspondingly the solenoid and TE mode field distributions. Then using Eqs. (23) and (24), we consider (for simplicity) TE and solenoid field distributions are identical to each other:

$$
\begin{aligned}
& B_{s}(z, r)= B_{s}\left[B_{s}(z, r) / B_{s}\right]=B_{s}\left\lfloor b_{0}(z)-r^{2} b^{\prime \prime}(z) / 4\right], \\
& b^{\prime \prime}(z)=\partial^{2} b_{0}(z) / \partial z^{2}, \\
& B_{\mathrm{TE}}(z, r, t)= B_{\mathrm{TE}}\left[B_{\mathrm{TE}}(z, r, t) / B_{\mathrm{TE}}\right] \\
&= B_{\mathrm{TE}}\left[b_{0}(z)-r^{2} b^{\prime \prime}(z) / 4-r^{2} k^{2} b_{0}(z) / 4\right] \\
& \times \cos (\omega t+\alpha),
\end{aligned}
$$

where $B_{s}, B_{\mathrm{TE}}$ is the maxima of solenoid and TE mode field distributions, respectively. Let us consider Eqs. (20) and (26) are completed; the integrating of Eq. (27) on the path length $z=0 \div L$ gives

$$
\begin{aligned}
\beta^{2} \gamma^{2}\left(\left.r^{\prime}\right|_{z=L}-\left.r^{\prime}\right|_{z=0}\right)= & -r\left(\frac{e}{2 m c}\right)^{2}\left(B_{s}^{2}+\frac{1}{2} B_{\mathrm{TE}}^{2}\right) \int b_{0}^{2}(z) d z+r \frac{2 \pi j_{0}}{I_{0} c} \frac{L}{\beta \gamma}+r^{3}\left(\frac{e}{2 m c}\right)^{2}\left(B_{s}^{2}+\frac{1}{2} B_{\mathrm{TE}}^{2}\right) \int b_{0}(z) b^{\prime \prime}(z) d z \\
& +r^{3}\left(\frac{e}{2 m c}\right)^{2} \frac{k^{2}}{4} B_{\mathrm{TE}}^{2} \int b_{0}^{2}(z) d z=0
\end{aligned}
$$

In order to get the radial independence in Eq. (30), the factors of $r$ and $r^{3}$ must be zero. This is possible if the values of $B_{s}, B_{\mathrm{TE}}$ meet the following requirements:

$$
\begin{gathered}
B_{\mathrm{TE}}^{2}\left[T^{2}\right]=4 \Lambda \frac{L j_{0}}{\beta \gamma} \frac{-\int b_{0}(z) b^{\prime \prime}(z) d z}{k^{2}\left[\int b_{0}^{2}(z) d z\right]^{2}}, \\
\Lambda=\frac{8 \pi c}{I_{0}(e / m)^{2}}=1.354 \times 10^{-17}\left[\frac{\mathrm{m} \mathrm{kg}^{2}}{\operatorname{coul}^{3} \mathrm{~s}^{2}}\right] \\
B_{s}^{2}\left[T^{2}\right]=\frac{L j_{0}}{\beta \gamma} \frac{\Lambda}{\int b_{0}^{2}(z) d z}\left(1+\frac{2 \int b_{0}(z) b^{\prime \prime}(z) d z}{k^{2} \int b_{0}^{2}(z) d z}\right) .
\end{gathered}
$$

If the beam current is $100 \mathrm{~A}$, beam radius is $20 \mathrm{~mm}, L=$ $1.5 \mathrm{~m}$, and $\omega / 2 \pi=2.47 \mathrm{GHz}$, we get $B_{\mathrm{TE}}=0.022 \mathrm{~T}$, $B_{s}=0.028 \mathrm{~T}$.

In all considered examples, the $B$ field amplitudes of TE modes have relatively small values. Also, these must be less for low particle energies as $B^{2} \sim \beta^{2} \gamma^{2}$ according to Eq. (18). Therefore, these TE cavities may be made of normal conducting materials. The rf power in the copper cavity of Fig. $10(E=3 \mathrm{MeV})$ is equal to $260 \mathrm{~kW}$ and maximal power density in the cavity wall is less than $250 \mathrm{~W} / \mathrm{cm}^{2}$. In this case the water cooling is possible.

We note that all TE cavity sizes can be widely scaled proportionally. In such a procedure, Eqs. (20) and (26) are left completed always. For the unchanged focusing strength, the dissipating power in the normal conducting cavity wall is left unchanged, i.e., the power density is changed inversely proportional to the scaling factor squared.

\section{TE MODE RESONANCES AND SELF-FOCUSING OF AN ELECTRON BEAM}

In Figs. 5 and 6, the Fourier transforms given in Eq. (20) are shown for different magnetic fields. If for a certain frequency $\omega_{0}$ the transform is zero, the energy transfer between the particles and the corresponding TE mode field vanishes also. In further chapters, we will discuss the converse case. The frequency of the TE mode is near the maximum of the Fourier transform. Then the energy transfer is sensitive to the phase of the rf field according to Eqs. (14) and (17).

For a beam without angular momentum and for a cavity without TE mode field, there always exist small TE mode field or beam angular pulse disturbances, which lead the energy transfer appeared, according to Eqs. (8) and (17). If the quality factor is high enough or dissipative power losses in the cavity are small enough then such modes can lead to an amplifying action. This is a quite distinct mechanism of the TE field self-excitation. The same effects exist in the problem of dipole modes self-excitation, which leads the breakup instability [16,17]. TE field selfexcitation only leads the beam focusing without the beam breaking up. 
The phase of the self-excited TE field by synchronic bunches must be determined by the condition that the energy particle-field transfer has a maximum. From Eq. (19) we obtain

$$
\alpha=-\Phi / 2=-\arg \left(\int_{-\infty}^{\infty} B^{2}(z) e^{j 2 \omega z / v_{0}} d z\right) / 2,
$$

and for the energy transfer Eq. (17) gets

$$
U \equiv \frac{\Delta W}{e}=\frac{e \omega \cdot r_{0}^{2}}{8 m \gamma_{0} v_{0}}\left|\int_{-\infty}^{\infty} B^{2}(z) e^{j 2 \omega z / v_{0}} d z\right|
$$

We consider that this maximal energy is transferred to the field of excited TE mode by a single particle synchronized with the TE field.

In the following we will analyze the interaction between the rf field of the TE mode and a pulsed electron beam in the absence of an external $\mathrm{rf}$ source. For this purpose, as a rule, the cavity is described by an equivalent circuit of an oscillatory contour excited by an external current source [18]. This excited voltage amplitude is $U=\Delta W / e$, the resonant frequency is $2 \omega$, and the quality factor is $2 Q$. This doubling follows from $2 \alpha$ double phase dependence in Eqs. (14) and (17). The quality factor and power losses $P$ of the equivalent circuit are given by equations

$$
Q=\frac{\omega \tau}{2}, \quad P=\omega \frac{J}{Q},
$$

where $J$ is TE field energy, and $\tau$ is the decay time of the cavity voltage.

For the current $I$, we assume that it is composed of bunches with the charge $q$ and the repetition frequency $\Omega$ so that $I=q \Omega /(2 \pi)$. This current excites the field $B(z)$ of Eq. (34). We would like to remark that, in contrast to previous considerations [19], the voltage $U$ and the impedance $R / Q$ are not parameters, which are determined by the cavity only. In the case of TE modes, they depend on the energy, field phase, amplitude $B_{\max }$ and $r$ of particle trajectories in the cavity also.

In order to give a numerical example, we have calculated $U_{0}$ by means of Eq. (34) as energy losses of particles in a tracking calculation using the ASTRA code [7] and the $\mathrm{TE}_{011}$ mode of the TESLA cell shown in Fig. 1. In this calculation, the bunch charge of $1 \mathrm{nC}$ is homogenously distributed in a cylinder with a radius of $2 \mathrm{~mm}$ and a length of $6 \mathrm{~mm}$. The particle energy is $3 \mathrm{MeV}\left(\gamma_{0} \sim 6\right)$. Space charge forces are included. Figure 12 shows the dependence of the voltage $U_{0}$ and the phase $\alpha$ from the amplitude $B_{\max }$ of the axis field $B(z)$. The phase $\alpha$ is obtained from the condition that $U_{0}$ has a maximum for each $B_{\max }$ value. TE field phase $\alpha$ of the cavity must be synchronized with the beam bunches. For this purpose, the electronic system of the phase control and the mechanical system of cavity deformation must be used.

$U_{0}$ can be quite well approximated by the polynomial of second degree

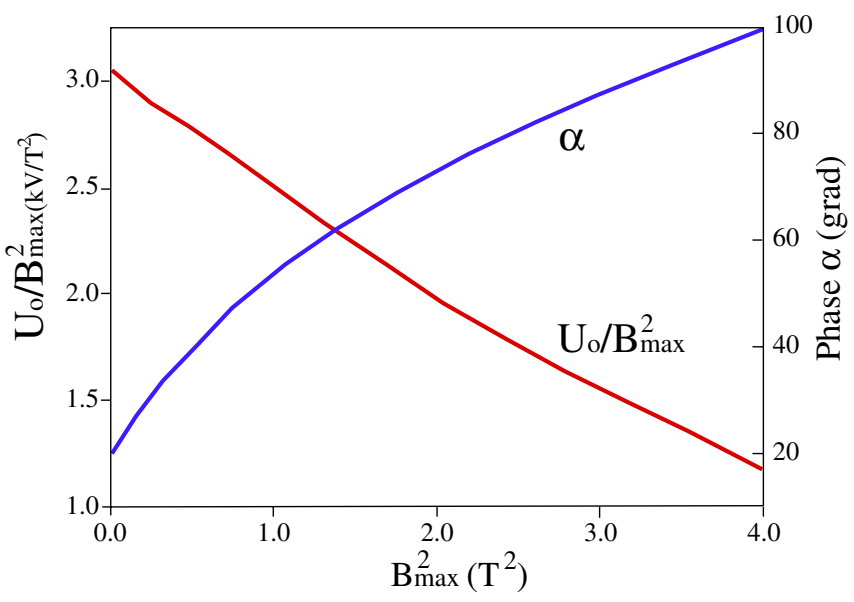

FIG. 12. (Color) $U_{0} / B_{\max }{ }^{2}$ and $\alpha$ as a function of $B_{\max }{ }^{2}$.

$$
\begin{aligned}
U_{0}[V] & =B_{\max }^{2}[T]\left(3054-616 B_{\max }^{2}[T]+36 B_{\max }^{4}[T]\right) \\
& \leq B_{\max }^{2} U_{0}^{\prime},
\end{aligned}
$$

where $U_{0}^{\prime}=3054$. For $B_{\max }<0.5 \mathrm{~T}$ the voltage is less than $1 \mathrm{kV}$.

Taking into account Eq. (34) we obtain the amplitude $U$ for other parameters of $r, \gamma, \omega$ instead of Eq. (36) calculated for $r_{o}=2 \mathrm{~mm}, \gamma_{0} \approx 6$, and $\omega_{0}=2 \pi \cdot 2.496 \times 10^{9}$ :

$$
U=U_{0} \cdot\left(r^{2} \omega / \gamma\right) /\left(r_{0}^{2} \omega_{0} / \gamma_{0}\right)=U_{0}\left(r^{2} \omega / \gamma\right) \times 10^{-4} .
$$

In the stationary case the energy transfer of the bunch to the rf field $U I$ is equal to the energy losses at the cavity surface [see Eq. (35)]. This is described by the equation [18]

$$
U I=\omega \cdot \frac{J}{Q}
$$

Using the notation $J=B_{\max }^{2} J^{\prime}$, where $J^{\prime}=138.4\left\lfloor\mathrm{~J} / \mathrm{T}^{2}\right\rfloor$ is the CLANS calculated factor for the TESLA cell, we obtain from Eqs. (36)-(38)

$$
I Q \geq \frac{J^{\prime}}{U_{0}^{\prime}} \frac{\gamma}{r^{2}} \times 10^{4}=450 \frac{\gamma}{r^{2}} .
$$

In TESLA cell cavity (see Fig. 1: $Q=8 \times 10^{9}, \omega=$ $2 \pi \cdot 2.496 \times 10^{9} \mathrm{~Hz}$ ), the threshold current of the beam is $I \geq 88 \mathrm{~mA}$. We can get the lower threshold current by one or two orders if we choose the low energy of the beam, large beam radius, and more compatible cavity shape. A special shape design of the cavity can minimize the characteristic value $J^{\prime} / U_{0}^{\prime}$, which enters Eq. (39) by 1 order of magnitude in comparison with the TESLA cell value.

For the excitation of this resonance by the beam current of a few tens mA, a large $Q$ value of $10^{8}-10^{9}$ is necessary. These values are reasonable for a superconducting cavity, but not reachable for a normal conducting one. 
The beam with the current of a few tens $\mathrm{mA}$ establishes its own magnetic field and focused itself. In this way, we have obtained an rf lens that is operated without an external power source. But the electronic system of phase control and mechanical system of the cavity tuning to the resonance must be used. Each bunch is focused with the same phase and amplitude. Therefore, this lens produces no additional occasional growth of the beam emittance.

\section{ENHANCEMENT OF THE FEL ACCEPTANCE IN THE THZ REGION}

The transverse acceptance of a FEL is limited by the $\left(90^{\circ}\right)$ phase slip condition [20]

$$
\left|\frac{1}{\lambda_{U}}-\frac{1}{2 \lambda \gamma^{2}}\left(1+\left\langle\gamma^{2} \beta_{\perp}^{2}\right\rangle\right)\right|<\frac{1}{4 L_{U}},
$$

where $L_{U}$ and $\lambda_{U}$ are the undulator parameters and $\beta_{\perp}$ is the electron velocity perpendicular to the beam axis. The electron motion inside the FEL can be described by a superposition of the oscillation with the wave number $2 \pi / \lambda_{U}$ and betatron oscillation with the wave number $k_{\beta}$. For a helical undulator with the field parameter $K$, one gets approximately [20]

$$
\left\langle\gamma^{2} \beta_{\perp}^{2}\right\rangle \approx K^{2}\left[1+\frac{1}{2}\left(\frac{2 \pi}{\lambda_{U}}\right)^{2} R_{m}^{2}\right] .
$$

The radius $R_{m}=\sqrt{X_{m}^{2}+Y_{m}^{2}}$ is defined by the amplitudes $X_{m}$ and $Y_{m}$ of the betatron oscillation in the $x$ and $y$ directions. These amplitudes depend only on the electron coordinates $x_{0}, x_{0}^{\prime}$ and $y_{0}, y_{0}^{\prime}$ at the entrance of the FEL and the wave number $k_{\beta}$ of the betatron oscillation, i.e.

$$
X_{m}^{2}=x_{0}^{2}+\left(x_{0}^{\prime} / k_{\beta}\right)^{2}, \quad Y_{m}^{2}=y_{0}^{2}+\left(y_{0}^{\prime} / k_{\beta}\right)^{2} .
$$

If the beam waist is placed in the middle of the undulator, we obtain $R_{m}^{2}=r_{0}^{2} \cdot\left(1+\frac{4}{\left(k_{\beta} L_{U}\right)^{2}}\right)$ with $r_{0}^{2}=x_{0}^{2}+y_{0}^{2}$. After this consideration, the acceptance condition of Eq. (40) is transformed to

$$
\begin{aligned}
& \left|\frac{1}{\lambda_{U}}-\frac{1}{2 \lambda \gamma^{2}}\left[1+K^{2}\left\{1+r_{0}^{2} \frac{1}{2}\left(\frac{2 \pi}{\lambda_{U}}\right)^{2}\left(1+\frac{4}{\left(k_{\beta} L_{U}\right)^{2}}\right)\right\}\right]\right| \\
& \quad<\frac{1}{4 L_{U}}
\end{aligned}
$$

and delivers the upper limit for the initial radius $r_{0}$ of the electrons.

It is evident that an appropriate radius dependence on the energy $\gamma$ cancels this limitation [21]. Neglecting terms of the order of $\left(\frac{r_{0}}{\lambda_{U}}\right)^{4}$ the radius dependence of $\gamma$ is given by

$$
\gamma=\gamma_{0}\left[1+r_{0}^{2} \frac{K^{2} \pi^{2}}{\lambda_{U}^{2}\left(1+K^{2}\right)}\left(1+\frac{4}{\left(k_{\beta} L_{U}\right)^{2}}\right)\right] .
$$

In this case, the wavelengths $\lambda$ are independent of the electron radius at the entrance of the undulator and all particles radiate with the same wavelength

$$
\lambda=\frac{\lambda_{U}}{2 \gamma_{0}^{2}}\left(1+K^{2}\right) .
$$

In the next step, we will discuss the possibility that the radius dependence of the beam energy given in Eq. (44) could be generated through the rf field of a TE mode. In this case, one has to accommodate the rf cavity directly before the undulator. The energy transfer from the rf field to the electron beam is given by Eq. (17). Neglecting the time dependence of the radius inside the cavity, the transferred energy is proportional to the square of the radius. Then the comparison of Eqs. (17) and (41) allows finding the condition, that the radius dependence on the FEL wavelength is compensated by a TE mode with the axis field $B(z)$ and the frequency $\omega$ :

$$
\begin{aligned}
\omega \int_{-\infty}^{\infty} B^{2}(c t) e^{j 2 \omega t+j 2 \alpha} d t= & 8\left(\frac{\gamma_{0} m c}{\lambda_{U} e}\right)^{2} \frac{K^{2} \pi^{2}}{1+K^{2}} \\
& \times\left(1+\frac{4}{\left(k_{\beta} L_{U}\right)^{2}}\right) .
\end{aligned}
$$

In this equation, the undulator parameters and the beam energy determine the strength of the magnetic axis field that is necessary to cancel the radius dependence of the FEL wavelength. Introducing the maximum axis field $B_{\max }$ from Eq. (46) gives

$$
B_{\max }[T]=0.0151 \frac{\gamma_{0}}{\sqrt{F} \lambda_{U}[m]} \frac{K}{\sqrt{1+K^{2}}} \sqrt{1+\frac{4}{\left(k_{\beta} L_{U}\right)^{2}}} .
$$

The parameter $F(\omega)=\omega\left|\int_{-\infty}^{\infty} \frac{B^{2}(c t)}{B_{\max }^{2}} \cdot e^{j 2 \omega t} d t\right| \leq 1.7$ is dimensionless. $F$ denote maximum with respect to $\omega$. This value is nonsensitive to the special shape of $B(z) / B_{\max }$. We have approximated this shape by the Gaussian function, quadratic function $1-(z / L)^{2}(|z|<L)$, and by the axis field of the pillbox cavity discussed in Sec. III. The value of $F$ remains to be within the limit $1.6<F<1.7$. Assuming $F=1.7, K=0.7$ and neglecting the term $\frac{4}{\left(k_{\beta} L_{U}\right)^{2}}$ in the root of Eq. (47), we obtain

$$
B_{\max }[T] \approx 0.00664 \frac{\gamma_{0}}{\lambda_{U}[m]} .
$$

For a superconducting cavity, $0.3 \mathrm{~T}$ is an achievable value of $B_{\max }$. Then an undulator period of $10 \mathrm{~cm}$ leads to $\gamma_{0}=4.52$ and a radiation wavelength $\lambda=3.6 \mathrm{~mm}$. The calculation of the parameter $F$ has been done for a single cell cavity. In the general case, one has to multiply the obtained value by the number of cavities. It can substantially enhance the FEL performances for wavelengths $\lambda \geq$ $1 \mathrm{~mm}$.

\section{CONCLUSION}

In a special shaped cavity cell, where the Fourier transform of the square of the axis $B$ field is zero, the magnetic 
field of a TE mode is equivalent to the non-time-dependent field of a static solenoid and can be used for the same intentions, e.g., for the focusing of continuous beams. Because of the distinction of its nonlinearities, the compensation of spherical aberrations is possible. A new derivation of $\mathrm{rf}$ resonance amplitudes provides the condition for the occurrence of TE mode resonances and the selffocusing of an electron beam. For large values of the mentioned Fourier transform, the TE mode field is able to cancel the radial acceptance limit of a helical FEL for a wavelength in the $\mathrm{THz}$ region. A quadruple TE mode should be able to overcome this limit also for planar radiation and shorter wavelength.

\section{ACKNOWLEDGMENTS}

The authors are thankful to T. Smith, R. Wuensch, and all the members of the Rossendorf rf group for many helpful discussions. Also thanks to V. Petrov for scientific discussions and other colleagues from BINP for interest in the work.

[1] D. Janssen et al., Nucl. Instrum. Methods Phys. Res., Sect. A 528, 305 (2004).

[2] J.D. Jackson, Classical Electrodynamics (Wiley, New York, 1998), 3rd ed.

[3] http://xfel.desy.de/tdr.

[4] D. G. Myakishev and V. P. Yakovlev, in Proceedings of the Particle Accelerator Conference, San Francisco, CA, 1991 (IEEE, Piscataway, NJ, 1991), Vol. 5, pp. 3002-3004.
[5] D. G. Myakishev and V. P. Yakovlev, in Proceedings of the Particle Accelerator Conference, New York, 1999 (IEEE, Piscataway, NJ, 1999), pp. 2775-2777.

[6] H. Busch, Z. Phys. 81, 974 (1926).

[7] K. Flottmann, ASTRA User's Manual, http://www.desy. de mpyflo/Astra_docomentation.

[8] S. J. Russel, Nucl. Instrum. Methods Phys. Res., Sect. A 507, 304 (2003).

[9] V. Volkov, K. Floettmann, and D. Janssen, in Proceedings of the Particle Accelerator Conference, Albuquerque, NM, 2007 (to be published).

[10] J.D. Lawson, The Physics of Charged-particle Beams (Clarendon Press, Oxford, 1977).

[11] L. Serafini, Nucl. Instrum. Methods Phys. Res., Sect. A 340, 40 (1994).

[12] J. Gao, Nucl. Instrum. Methods Phys. Res., Sect. A 304, 353 (1991).

[13] J. Zach, Nucl. Instrum. Methods Phys. Res., Sect. A 298, 255 (1990).

[14] K. J. Harker, Trans. Electron Devices 2, 4 (1955).

[15] A. M. Glogston, J. Appl. Phys. 42, 4 (1954).

[16] W. K. H. Panofsky and M. Bander, Rev. Sci. Instrum. 39, 206 (1968).

[17] V. K. Neil and R. K. Cooper, Part. Accel. 1, 111 (1970).

[18] R.E. Collin, Foundations of Microwave Engineering (IEEE Press, New York, 1992).

[19] V. Volkov and D. Janssen, in Proceedings of the FEL Conference 2007, Novosibirsk, Russia, see http:// accelconf.web.cern.ch/AccelConf/f07/INDEX.HTM.

[20] T. Smith and J. M. J. Madey, Appl. Phys. B 27, 195 (1982).

[21] T. Smith, in ELBE Workshop 2007, Dresden, Germany (unpublished). 